\title{
An Observational Study on 100 Patients with Polycystic Ovarian Syndrome (PCOS)
}

\author{
Khaleda Khanam ${ }^{1}$, Mimi Parvin ${ }^{2}$ \\ Received: March 18, 2014 Accepted: June 18, 2014
}

\begin{abstract}
Background: Women with polycystic ovarian syndrome (PCOS) have chronic anovulation and androgen excess not attributable to another cause. The fundamental pathophysiologic defect is unknown. Defects in LH secretion, LH/FSH ratio, amplitude of LH pulsations have been described; but the prevalence of these defects is not still clearly determined. Objective: To review the variable clinical presentations of polycystic ovarian syndrome. Materials and Methods: This observational study was carried out in Combined Military Hospitals of Jessore, Rangpur and Ghatail during November 2008 to June 2013. One hundred patients attending Gynaecology Outpatient Department (GOPD) having at least two of the following criteria hyperandrogenism, chronic oligo- or anovulation and ultrasonographic findings were selected. In all selected women LH and FSH serum levels were determined and LH/FSH ratios were calculated. Body mass index (BMI) was measured and was scored to classify their state of obesity. The collected data were compiled and arranged in tables and were subjected to analysis. Results: Most of the patients (92\%) were 20-30 years old. Chief complaint of the patients was infertility, either primary (72\%) or secondary (28\%). Eighty percent women had menstrual irregularities, 30\% were hirsute, $71 \%$ cases were overweight and $17 \%$ were obese. On pelvic ultrasonogram polycystic ovaries were found in $20 \%$ cases and $80 \%$ had normal ovaries. Thirty percent patients had LH/FSH ratio between 2.1-2.9, $32 \%$ had $\geqslant 3$ and it was found normal in $38 \%$ of cases. Conclusion: PCOS cannot be diagnosed by a single clinical or laboratory finding. The diagnostic approach should be based largely on history and physical examination.
\end{abstract}

Key words: Polycystic ovary syndrome; LH/FSH ratio; Infertility; Obesity

J Enam Med Col 2014; 4(3): 156-160

\section{Introduction}

Polycystic ovarian syndrome (PCOS) has also been known by the name Stein-Leventhal Syndrome, and includes a multisystem presentation, having its effects on the skin, hair, body weight and endocrine and reproductive systems. ${ }^{1}$ It is said to affect up to $10 \%$ of women of reproductive age, and up to $90 \%$ of women with irregular menstrual cycles., ${ }^{1,2}$ Variance in prevalence among populations may reflect the effect of ethnic origin, race, and other environmental factors on the phenotype. $^{3}$
PCOS was first recognised as a medical disease or syndrome in 1845 in France. Its most distinctive sign is the pathological appearance of sclerocystic changes on the larger than average ovaries which appeared to have a thick, shiny, white coating overlying many rows of cysts on the surface of the ovary. These ovarian changes give PCOS its name. In 1990 a National Institute of Health conference decided the two most consistent elements which comprise the disorder of PCOS that include presence of hyperandrogenism and chronic

1. Classified Specialist, Department of Obstetrics and Gynaecology, Combined Military Hospital, Shaheed Salahuddin Cantonment, Ghatail, Tangail

2. Classified Specialist, Department of Pathology, Combined Military Hospital, Shaheed Salahuddin Cantonment, Ghatail, Tangail

Correspondence Khaleda Khanam,Email: khaledashah@yahoo.com 
oligo- or anovulation, with exclusion of other causes of hyperandrogenism such as adult onset congenital adrenal hyperplasia, hyperprolactinaemia and androgen secreting neoplasms. ${ }^{4}$ A consensus conference held in Rotterdam, in May 2003, sponsored by the European Society of Human Reproduction and Embryology (ESHRE) and American Society for Reproductive Medicine (ASRM) not only agreed on the appropriateness of including ultrasound morphology of the ovaries as a further potential criterion to define the PCOS but also established that at least two of the following criteria are sufficient for the diagnosis: oligoor anovulation, clinical and/or biochemical signs of hyperandrogenism and polycystic ovaries on ultrasound. ${ }^{5}$ The pathophysiology of PCOS may have a genetic component although it can be suggested that the main factors responsible for the increasing prevalence of PCOS are related to the influence of the environment, including dietary habits, behaviour and other still undefined factors. ${ }^{1}$ The clinical features of PCOS are heterogeneous and may change throughout the lifespan, starting from adolescence to postmenopausal age. ${ }^{6}$ This is largely dependent on the influence of obesity and metabolic syndrome which consistently affect most women with PCOS. ${ }^{7}$ This represents an important factor in the evaluation of the PCOS throughout life and implies that the PCOS by itself may not be a hyperandrogenic disorder exclusively restricted and relevant to young and fertile aged women but may also have some health implications later in life. Whereas hyperandrogenism and menstrual irregularities represent the major complaints in young women with the PCOS, symptoms related to androgen excess, oligomenorrhoea or amenorrhoea and, particularly, infertility are the main complaints of adult women with PCOS during the reproductive age. Obesity has an important impact on the severity of these manifestations in proportion to its degree and particularly in the presence of the abdominal phenotype. ${ }^{5}$ In addition, there is consistent evidence that it renders affected women more susceptible to develop type 2 diabetes, with some differences in the prevalence rates among countries and, potentially, in favouring the development of cardiovascular diseases. ${ }^{1}$ Defects of gonadotrophin secretion, including an elevated LH level, elevated LH to FSH ratio, and an increased frequency and amplitude of LH pulsations have been described, but the prevalence of these defects in a large, unbiased population of PCOS patients has not been determined. The present study was carried out to evaluate the characteristics and laboratory examination (available at Armed Forces Institute of Pathology, Dhaka Cantonment) findings of PCOS patients attending in different Combined Military Hospitals $(\mathrm{CMH})$ in Bangladesh.

\section{Materials and Methods}

This cross sectional observational study was carried out in Combined Military Hospitals (CMH) of Jessore, Rangpur and Ghatail during November 2008 to June 2013. The data were collected in the preformed data collection sheet for this study which included age, menstrual history including regularity/irregularity of cycle, length of cycle, obstetric (degree of fertility) and medical history, body mass index, acne and hirsutism. One hundred patients attending Gynaecology Outpatient Department (GOPD) were selected. Patients having at least two of the following criteria hyperandrogenism, chronic oligo- or anovulation and ultrasonographic findings were included in the study. Patients suffering from thyroid dysfunction and hyperprolactinaemia (diagnosed biochemically), Cushing's syndrome and virilising tumours (diagnosed clinically) were excluded from the study. In all selected women LH and FSH serum levels were determined and LH/FSH ratios were calculated. LH/FSH ratio $>2$ was considered abnormal. Because of the pulsatile nature of their release, a single test may fail to detect an increased $\mathrm{LH} / \mathrm{FSH}$ ratio. So we collected two fasting blood specimens, first at cycle day 4-6 from those with regular cycles or any day from those with amenorrhoea or completely irregular cycles and a second specimen was obtained 3-4 days later according to availability of patients. The LH and FSH were measured by electrochemiluminescence technique in COBAS autoanalyzer using reagents manufactured by Roche Diagnostics and quality control was done by preci-control 1 and 2, then mean LH/FSH ratio was obtained. Body mass index (BMI) of all subjects was calculated according to WHO and International Obesity Task Force classification. ${ }^{8}$ The collected data were compiled and analysed. Approval for this study was obtained from the institutions and informed consents were taken from all the study subjects.

\section{Results}

The characteristics (age, BMI, menstrual status, hirsutism, fertility status and LH/FSH ratio) of women included in this study are shown in Table I and LH/FSH ratio based on BMI is shown in Table II. 
Majority of our subjects (92\%) were between 20-30 years of age. Chief complaint of the patients was infertility, either primary (72\%) or secondary (28\%). Majority of women $(80 \%)$ had menstrual irregularities, only $20 \%$ had regular menses.

Thirty percent women were found hirsute while $70 \%$ were nonhirsute, 54\% cases were overweight, 34\% obese and $12 \%$ were of normal weight. On pelvic ultrasonogram, polycystic ovaries were found in $20 \%$ cases and $80 \%$ had normal ovaries. Thirty percent patients had LH/FSH ratio between 2.1-2.9, 32\% had $\geqslant 3$ and it was found normal in $38 \%$ of cases.

In overweight and obese (BMI $\geqslant 25$ ) category there were 88 patients, with high $\mathrm{LH} / \mathrm{FSH}$ ratio in 52 and in normal weight category $(\mathrm{BMI}<25)$ there were 12 patients, with high $\mathrm{LH} / \mathrm{FSH}$ ratio in 10 subjects (Table II).

Table I: Age distribution, BMI, menstrual cycle, types of infertility, presence of hirsutism, ultrasonographic findings and LH/FSH ratio of study subjects $(n=100)$

\begin{tabular}{|c|c|c|}
\hline Variables & Frequency & Percentage \\
\hline Age in years & & \\
\hline $20-25$ & 52 & 52 \\
\hline $26-30$ & 40 & 40 \\
\hline$>30$ & 08 & 08 \\
\hline \multicolumn{3}{|l|}{ BMI } \\
\hline$<25$ & 12 & 12 \\
\hline $25-29.9$ & 54 & 54 \\
\hline$\geqslant 30$ & 34 & 34 \\
\hline \multicolumn{3}{|l|}{ Menstrual cycle } \\
\hline Regular & 20 & 20 \\
\hline Irregular & 80 & 80 \\
\hline \multicolumn{3}{|l|}{ Types of infertility } \\
\hline Primary & 72 & 72 \\
\hline Secondary & 28 & 28 \\
\hline \multicolumn{3}{|l|}{ Hirsutism } \\
\hline Present & 30 & 30 \\
\hline Absent & 70 & 70 \\
\hline \multicolumn{3}{|l|}{ Ultrasonogram findings } \\
\hline Normal ovaries & 80 & 80 \\
\hline Polycystic ovaries & 20 & 20 \\
\hline \multicolumn{3}{|l|}{ LH/FSH ratio } \\
\hline$<1$ & 18 & 18 \\
\hline $1-2$ & 20 & 20 \\
\hline $2.1-2.9$ & 30 & 30 \\
\hline$\geqslant 3$ & 32 & 32 \\
\hline
\end{tabular}

Table II: Distribution of study subjects with BMI and LH/FSH ratio $(n=100)$

\begin{tabular}{|l|c|c|c|c|c|}
\hline \multirow{2}{*}{ BMI } & \multicolumn{4}{|c|}{ LH/FSH ratio } & Total \\
\hline & Up to 1 & $1-2$ & $2.1-2.9$ & $\geqslant 3$ & \\
\hline$<25$ & 01 & 01 & 09 & 01 & 12 \\
\hline $25-29.9$ & 05 & 05 & 14 & 30 & 54 \\
\hline$\geqslant 30$ & 10 & 16 & 06 & 02 & 34 \\
\hline
\end{tabular}

\section{Discussion}

Polycystic ovary syndrome is a subject of continuous studies concerning pathogenesis, diagnostic methods, and therapeutic procedures. Now-a-days attention is focused on the role of insulin resistance, hyperinsulinaemia and insulin-like growth factor I (IGF-I) in development of syndrome. Abnormality of the hypothalamic-pituitary-ovarian or adrenal axis has been implicated in PCOS. Disturbance in the pulsatility of gonadotrophin releasing hormone $(\mathrm{GnRH})$ results in the relative increase in LH to FSH ratio. An abnormal feedback mechanism by ovarian oestrogen is blamed to play role in this discriminated increase in LH release. As a result of this derangement the ratio between LH and FSH levels which is normally around 2 to 1 , becomes reversed and sometimes even more (2 or 3 to 1) in approximately $60 \%$ of the patients with PCOS. ${ }^{9} \mathrm{At}$ the end of 1980s LH/FSH ratio was still perceived as a "gold standard" for diagnosis of PCOS, and the coexistence of insulin resistance and hyperinsulinaemia was only emerging as a potential pathogenic factor. The overproduction of $\mathrm{LH}$ and consequently the incorrect LH/FSH ratio is now-a-days considered not to be a characteristic attribute of all PCOS patients. In this study elevated gonadotrophin ratio was found in $62 \%$ cases which differs from the findings of Banaszewska et al who found the incidence to be $45.4 \%$; but some studies assess the incidence of elevated LH/FSH ratio at even $94 \% .{ }^{10}$ Our study differed from Banaszewska et al because their patients of distinct subgroup might have increased adrenal androgenic activity.

In this study we observed that there is heterogeneity in the presentation of the patients with PCOS. It is emphasised that PCOS is a syndrome with no significantly single diagnostic criterion (such as hyperandrogenism or polycystic ovaries) which is sufficient for clinical diagnosis. ${ }^{5}$ According to National Institute of Health-National Institute of Child Health 
and Human Development (NIH-NICHHD) criteria for diagnosis of PCOS (set in 1990), USG was not mandatory. ${ }^{11}$ Western data showed that around $50 \%$ of biochemically diagnosed PCOS had fulfilled USG criteria. ${ }^{12}$ Zawadski et $\mathrm{al}^{4}$ claimed that, polycystic morphology is consistent with, but not essential for diagnosis of the syndrome as we have found in our cases where $80 \%$ had normal ovaries. These changes, however, can be present in women who are endocrinologically normal. Thus, the ovarian morphologic change must be distinguished from the endocrine syndrome of hyperandrogenism and anovulation. ${ }^{9}$ USG is sensitive but not specific for diagnosis of PCOS. ${ }^{13}$ One disadvantage of the study was that we could not advise transvaginal sonography (TVS) due to reluctance of the participants.

The clinical consequence of chronic anovulation is some form of menstrual irregularity - oligomenorrhoea (menses every 6 weeks to 6 months), amenorrhoea or dysfunctional uterine bleeding. Infertility may be the presenting symptom of the anovulation. ${ }^{9}$ In our study most of the women seeking physicians' advice were due to infertility which is one of the common problems in married women in our society. Our study is similar to the study of Anwary et $\mathrm{al}^{14}$ in which infertility was found in $90 \%$ subjects. Out of the 100 women included in this study $80 \%$ had complained of irregular menstrual cycle and $20 \%$ had normal cycle which is similar to the study conducted by Anwary et al. ${ }^{14}$

Hirsutism was present in 30\% subjects, which differed from the study of Alnakash et $\mathrm{al}^{9}$ and Anwary et all4 as they have included peri-pubertal age when hirsutism draws more attention than menstrual irregularity and fertility. In their studies hirsutism was present in $64.49 \%$ and $50 \%$ cases respectively. Majority of our study subjects (92\%) belonged to the age group of 20 to 30 years, supporting the fact that PCOS are more prevalent in youngers than older women. It is probably due to a physiological decline of the follicular cohort leading to normalised ovarian ultrasonographic appearance with advancing age. ${ }^{9}$

In this study, $88 \%$ of the participants were overweight or obese $(\mathrm{BMI} \geqslant 25)$; this is similar to the findings of Pasquali et al ${ }^{15}$ and Kiddy et al. ${ }^{16}$ They found that about $50 \%$ and $35 \%$ (total $85 \%$ ) of the women with PCOS were obese and overweight respectively. But findings in our study differed from that of Alnakash et al. ${ }^{9}$ The explanation for higher frequency of obesity and overweight may be attributed to the food habits, lack of exercise in Bangladeshi women. Obesity frequently complicates polycystic ovarian syndrome but is not a defining characteristic. ${ }^{17}$

The traditional belief is that obesity plays a serious role in the pathophysiology of PCOS, but the puzzling fact is that not all PCOS women are obese. Moreover, not every women with PCOS has an abnormal LH/FSH ratio and hormonal and biochemical changes suggestive of this disease. This diversity in disease criteria inspired us to think about measuring correlations between disease manifestations and to answer the question "does higher BMI necessarily indicate a higher LH/FSH ratio". Our study shows that nearly $60 \%$ overweight and obese $(\mathrm{BMI} \geqslant 25)$ subjects had high $\mathrm{LH} / \mathrm{FSH}$ ratio.

PCOS is a very heterogeneous disorder of different phenotypes. No single clinical or laboratory finding can be a characteristic attribute to diagnose PCOS. The diagnostic approach should be based largely on history and physical examination, thus avoiding numerous laboratory tests that do not contribute to clinical management. Our results may provide an answer to the question raised before disapproving the traditional concept of disease which regard that heavier the patient is, the high is the LH/FSH ratio, hirsutism, infertility or the worse is the disease manifestation.

\section{References}

1. Ehrmann DA. Polycystic ovary syndrome. N Engl J Med 2005; 352(12): 1223-1236.

2. Homburg R. Polycystic ovary syndrome. Best Practice \& Research Clinical Obstetrics \& Gynaecology 2008; 22(2): 261-274.

3. Kauffman RP, Baker VM, DiMarino P, Gimpel T, Castracane VD. Polycystic ovarian syndrome and insulin resistance in white and Mexican American women: a comparison of two distinct populations. Am J Obstet Gynecol 2002; 187: 1362-1369.

4. Zawadski JK, Dunaif A. Diagnostic criteria for polycystic ovary syndrome; towards a rational approach. In: Dunaif A, Givens JR, Haseltine F (eds). Polycystic ovary syndrome. Boston: Blackwell Scientific, 1992: 377-384.

5. The Rotterdam ESHRE/ASRM sponsored PCOS consensus workshop group. Revised 2003 consensus on diagnostic criteria and long term health risks related to polycystic ovary syndrome (PCOS). Hum Reprod 2004; 19(1): 41-47. 
6. Pasquali R, Gambineri A.PCOS: a multifaceted disease from adolescence to adult age. Ann NY Acad Sci 2006; 1092(1): 158-174.

7. Gambineri A, Pelusi C, Vincennati V, Pagotto U, Pasquali R. Obesity and the polycystic ovary syndrome. Int J Obes Rel Metab Disord 2002; 26: 883-896.

8. Lobstein T, Rigby N, Leach R. International Obesity Task Force EU Platform briefing paper prepared in collaboration with the European Association for the Study of Obesity. Available at: http://ec.europa.eu/health/ ph_determinants/ life_style/nutrition/documents/iotf_en.pdf. Accessed June 2014 .

9. Alnakash AH, Al-Tae'e NK. Polycystic ovary syndrome: the correlation between the LH/FSH ratio and disease manifestation. Middle East Fertility Society Journal 2007; 12: $35-40$.

10. Banaszewaka B, Spaczyñski RZ, Pelesz M, Pawelczyk L. Incidence of elevated LH/FSH ratio in polycystic ovary syndrome women with normo- and hyperinsulinemia 2003; 48: 131-134.

11. Asncion M, Calvo RM, San Milan JL, Millan JL, Sancho J, Avila $\mathrm{S}$ et al. A prospective study of the prevalence of the
PCOS in unselected Caucasian women from Spain. J Clin Endocrinol Metabol 2000; 85: 2434-2438.

12. Khan MI, Klachko DM. Polycystic ovarian syndrome. Available at: www.emedicine.com $/ \mathrm{med} /$ topic2173.htm. Accessed June 2014

13. Dipankar B, Kumar MS, Satinath M, Mamata P. Clinical correlation with biochemical status in polycystic ovary syndrome. J Obstet Gynecol India 2005; 55: 67-71.

14. Anwary SA, Alfazzaman M, Begum N. A clinical study on PCOS patients in a tertiary hospital. Medicine today 2009; 22: 34-37.

15. Pasquali R, Vicennate V. Influence of weight and distribution of adipose tissue in functional hyperandrogenism. Cotracept-Fertil-Sex. 1998; 26: 372-375.

16. Kiddy DS, Sharp PS, White DM. Difference in clinical and endocrine features between obese and nonobese subjects with polycystic ovarian syndrome: an analysis of 263 consecutive cases. Clin Endocrinol Oxf 1990; 32: 213-220.

17. Guzick DS. Polycystic ovary syndrome. Obstet Gynecol 2004; 103(1): 181-193. 